\section{Global T waves inversion and QT prolongation. An uncommon presentation of acute pulmonary embolism}

\author{
Simone Savastano, ${ }^{1}$ Martina Querio, ${ }^{2}$ \\ Ernesto Anesi, ${ }^{3}$ Rita Camporotondo ${ }^{4}$ \\ 'Division of Cardiology, Fondazione \\ IRCCS Policlinico San Matteo, Pavia; \\ 2School of Cardiovascular Disease, \\ University of Pavia; 3Emergency \\ Department, Fondazione IRCCS \\ Policlinico San Matteo, Pavia; ${ }^{4}$ Intensive \\ Coronary Unit, Fondazione IRCCS \\ Policlinico San Matteo, Pavia, Italy
}

\begin{abstract}
This is the case of a man presenting to the emergency department for dyspnea. Despite a very common symptom he presented an uncommon twelve leads electrocardiogram (ECG). At a first glance it could have suggested an acute coronary syndrome, a Takotsubo cardiomyopathy or a hypertrophic cardiomyopathy. However the further investigations showed an acute pulmonary embolism (APE) whose pre-test probability was low with a Wells score of 0 and a Geneva simplified score of 1 . Negative $T$ waves have been described in APE, however, such a morphology associated with QT prolongation is a very rare presentation. This case confirms how the diagnosis of APE could be often insidious representing a challenge for the emergency physician.
\end{abstract}

\section{Case Report}

This is the case of a 82 -year-old male who presented to the emergency department of our Hospital after four days of exertional dyspnea. He used to lead an active life until the onset of symptoms. Dyspnea appeared suddenly with a constant progression to ever-smaller efforts. His past medical history was negative, with the exception of arterial hypertension. No past cardiac diseases or other comorbidities were known. On physical examination lung and heart sounds were clear with a systolic murmur in centrum cordis; abdomen examitation's findings were negative, no peripheral edema or jugular vein distension were detected. Laboratory tests showed a normal complete blood count, serum creatinine was 1.82 $\mathrm{mg} / \mathrm{dL}$, cardiac troponine I was $0.06 \mathrm{ng} / \mathrm{mL}$
(URL $0,04 \mathrm{ng} / \mathrm{mL}$ ). Figure 1 shows the ECG at admission.

The ECG shows a regular sinus rhythm at 66 beats per minute, a normal atrio-ventricular conduction, a normal QRS duration and global large and symmetrical $\mathrm{T}$ waves inversion ( $\max 9 \mathrm{~mm}$ in $\mathrm{V} 3$ ) with a QT prolongation $(\mathrm{QT} / \mathrm{QTc}=600 / 632 \mathrm{~ms})$.

Despite a very low pretest probability with a Wells score of 0 and a Geneva simplified score of 1 the echocardiographycal examination showed a right ventricle dilatation with reduced systolic function and systolic compression of the left ventricle which showed a preserved systolic function.

A compression ultrasound examination of the lower limbs was provided outlining a deep venous thrombosis involving the left femoral and popliteal veins. The arterial blood gas test showed hypoxia $(\mathrm{PaO} 2=$ $52.9 \mathrm{mmHg})$ and hypocapnia $(\mathrm{PaCO} 2=24$ $\mathrm{mmHg}$ ), potassium level was normal (4.6 $\mathrm{mmol} / \mathrm{L})$; the D-dimer DD10 was 12105 $\mu \mathrm{g} / \mathrm{L}$ (URL $500 \mu \mathrm{g} / \mathrm{L}$ ). Finally a chest CT scan confirmed the diagnosis of massive pulmonary embolism (Figure 2).

\section{Discussion}

Negative $T$ waves have been described in many clinical conditions such as acute coronary syndromes, Takotsubo cardiomyopathy, hypertrophic cardiomyopathy, acute cerebrovascular events, ionic disorders and acute pulmonary embolism (APE). The first description of negative T waves in APE was by Lowe et al. ${ }^{1}$ in 1938 . A case series by Ferrari et al. ${ }^{2}$ published in 1997 analyzed the different ECG manifestations of APE and it was pointed out that $T$ wave inversion in the anterior leads may be present in about $60 \%$ of the patients. Usually this presentation suggests a worse prognosis and can appear some days after the onset of symptoms. A more recent paper $^{3}$ sought for differences among $\mathrm{T}$ waves morphology occurring in acute coronary syndromes, in Takotsubo cardiomyopathy and in APE. In APE T waves usually remain positive in I, aVL (as in our case) and only in few cases $\mathrm{T}$ waves may be negative in V5, V6 and II. In the same paper the maximal $\mathrm{T}$ wav negativity was also compared and it was shown that in APE T waves were less negative than in the other two conditions with a maximal negativity of $3.4 \mathrm{~mm}$.

In our case $\mathrm{T}$ wave inversion was in some ways atypical for APE as T waves were negative also in II e V5 and mostly in V6 with a maximal negativity of about 9 $\mathrm{mm}$; there was no sinus tachycardia also frequent in APE, and the QT interval was prolonged.
Correspondence: Simone Savastano, Division of Cardiology, Fondazione IRCCS Policlinico San Matteo, Piazzale Golgi, 27100 Pavia, Italy.

Tel.: +39.0382.501590 - Fax: +39.0382.503161.

E-mail: s.savastano@smatteo.pv.it

Key words: ECG, pulmonary embolism, $\mathrm{T}$ waves.

Contributions: SS, cardiologist, evaluated the patients in the emergency department; MQ, fellow in cardiology, was present during the evaluation in the emergency department and recovered the material for the drafting of the clinical case; EA, physician, took care of the patient in the emergency department; RC, cardiologist, took care of the patient in the intensive coronary unit.

Conflict of interest: the authors declare no potential conflict of interest.

Received for publication: 15 October 2017. Revision received: 12 January 2018. Accepted for publication: 20 February 2018.

This work is licensed under a Creative Commons Attribution 4.0 License (by-nc 4.0).

(C) Copyright S. Savastano et al., 2017 Licensee PAGEPress, Italy

Emergency Care Journal 2017; 13:7144

doi:10.4081/ecj.2017.7144

The underlying mechanism of $\mathrm{T}$ waves inversion in APE is not completely known and some theories have been proposed. The first and the most accredited is the coronary insufficiency due to the decreased perfusion of the right ventricle caused by the increase of ventricular pressures and by the increase of afterload. Another possible explanation may be the effect of an increased release of catecholamine due to hypoxia and hypotension. The most recent theory lies on the potential effect of histamine release. The suffering lung tissue may release histamine which can induce myocardial ischemia and coronary vasospasm. From our perspective it appears quite difficult to find out a single mechanism which can justify the ECG pattern. The most likely hypothesis is a combination of the various mechanisms described above. In particular the prolongation of the QT interval suggest an underlying ischemia. Of course a previous ECG could have been interesting but it was not available.

\section{Conclusions}

In conclusion the current ECG with its atypical elements provides the occasion to meditate on the diagnosis of APE which is 


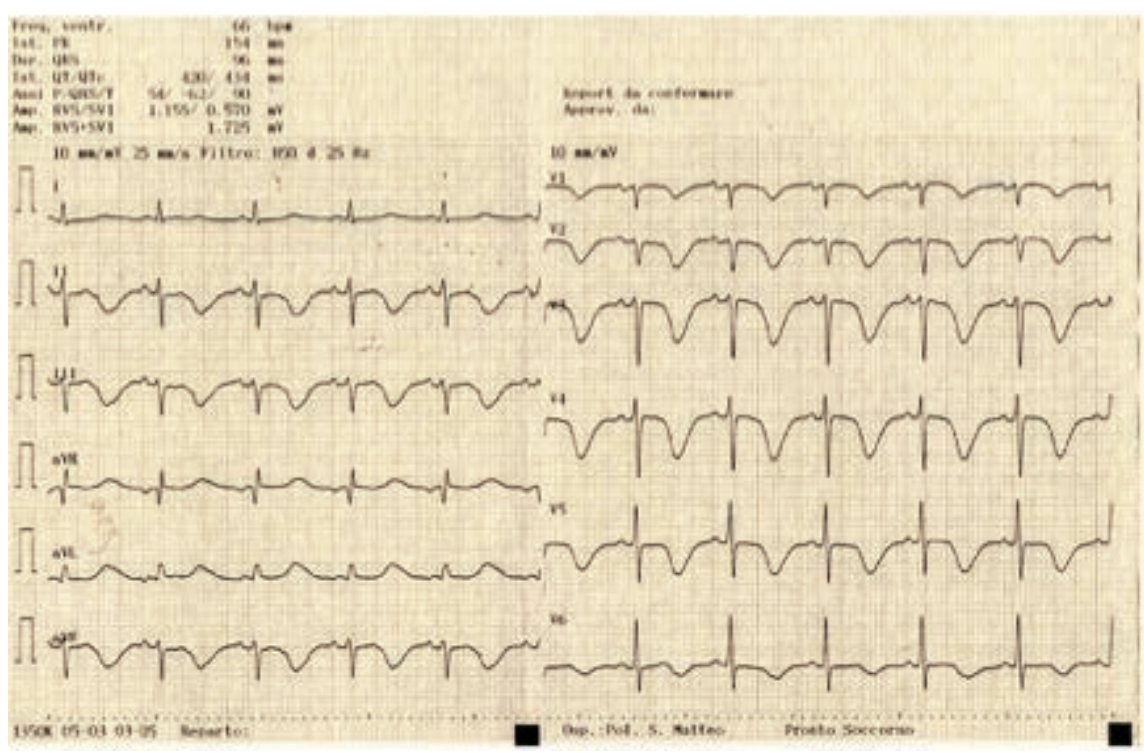

Figure 1. ECG at admission showing regular sinus rhythm, normal atrio-ventricular conduction and giant, global symmetrical $\mathrm{T}$ waves inversion and $\mathrm{QT}$ prolongation.

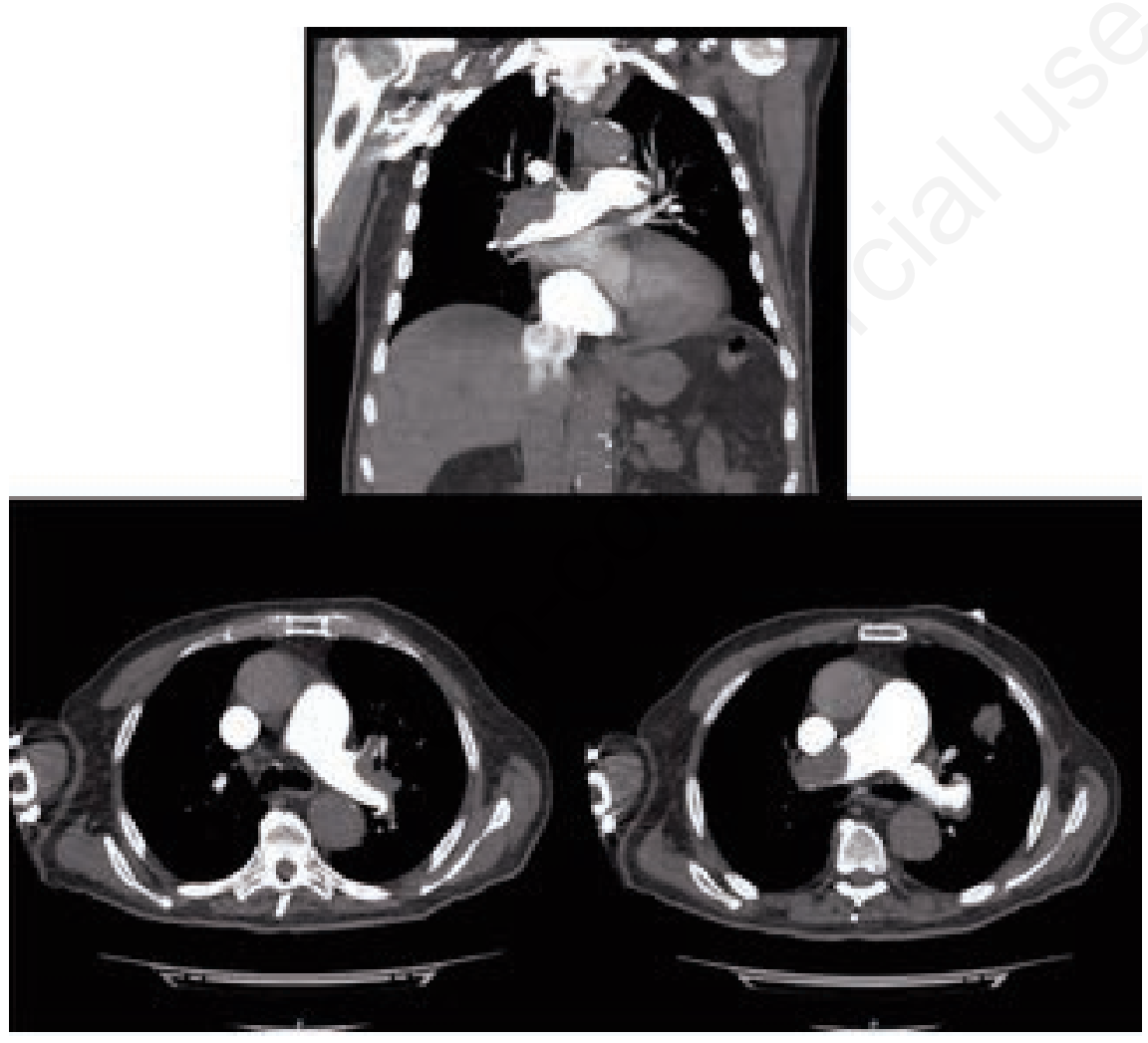

Figure 2. Chest CT scan images outlining a subocclusive thrombosis of the right pulmonary artery and of the superior branch of the left pulmonary artery. very often difficult and insidious representing a challenge for the emergency physician.

\section{References}

1. Lowe WS, Brugler GW, Winslow N Electrocardiographic studies of clinical and experimental pulmonary emobolisation. Ann Intern Med 1938;11:210923.

2. Ferrari E, Imbert A, Chevalier $\mathrm{T}$, et al. The ECG in pulmonary embolism. Predictive value of negative $\mathrm{T}$ waves in precordial leads 80 case reports. Chest 1997;111:537-43.

3. Kosuge M, Ebina T, Hibi K, et al. Differences in negative $\mathrm{T}$ waves among acute coronary syndrome, acute pulmonary embolism, and Takotsubo cardiomyopathy. Eur Heart J Acute Cardiovasc Care 2012;1:349-57. 